\title{
Ética y Responsabilidad Social en las alianzas estratégicas. El caso de las alianzas entre entidades lucrativas y no lucrativas
}

\author{
Carmen Talavera Avelino ${ }^{1}$ y Joan Ramon Sanchis Palacio ${ }^{2}[$
}

Recibido: 31 de julio de 2020 / Aceptado: 21 de octubre de 2020 / Publicado: 18 de febrero de 2021

Resumen. Las alianzas estratégicas han sido herramientas utilizadas por las organizaciones durante años para garantizar su desarrollo empresarial. En las últimas décadas, las demandas más exigentes de la sociedad han propiciado la necesidad de acuerdos de cooperación destinados a la creación de valor no únicamente para los socios implicados, sino también para los diferentes actores de la comunidad. Con esta finalidad, en el entorno actual se lleva a cabo un mayor número de alianzas estratégica entre entidades lucrativas y sin ánimo de lucro. Éstas no solo propician un impacto positivo para los socios a través de una mejora de su imagen o reputación, sino que también son propulsoras del desarrollo social y económico de aquellas áreas en las que se establecen u operan. A través de este tipo de acuerdos de cooperación, las organizaciones son capaces de crear valor tanto económico como social, asegurando de esta manera su supervivencia y el beneficio de los diferentes agentes de la comunidad en la que se encuentran. Los comportamientos éticos y las políticas de responsabilidad social favorecen la obtención de ventajas competitivas; y estos son posibles a través de las alianzas entre entidades lucrativas y no lucrativas. A través del estudio de casos de alianzas entre empresas lucrativas y entidades no lucrativas como Grupo Vips-Fundacion Hazlo Posible y Danone Foods-Grameen Bank se analizan los motivos por los cuales se llevan a cabo este tipo de alianzas, así como sus implicaciones y beneficios, tanto para las empresas socias como para las comunidades en las que operan. En el trabajo se analizan estos dos casos y se realiza también un análisis comparativo entre los dos con el objeto de establecer unas pautas de comportamiento comunes en este tipo de alianzas.

Palabras clave: Alianzas estratégicas; Ética; Responsabilidad Social; Valor Compartido; Entidades no lucrativas. Claves Econlit: D74; L31; M14.

\section{[en] Ethics and Social Responsibility in strategic alliances. The case of alliances between for-profit and non-profit organizations}

\begin{abstract}
Strategic alliances have been tools used by organizations for years to guarantee their business development. In recent decades, the greater demands of society have led to the need for cooperation agreements aimed at creating value not only for the partners involved, but also for the different actors in the community. To this end, in the current environment, a substantial number of strategic alliances between for-profit and non-profit entities are being carried out. This phenomenon not only promotes a positive impact for the partners involved through an improvement in their image or reputation, but they are also promoters of the social and economic development of those areas in which they are established or operate. Through this type of cooperation agreements, organizations are able to create both economic and social value, thus ensuring their survival and the benefit of the different agents of the community in which they are located. Ethical behaviors and social responsibility policies favor obtaining competitive advantages; and these are possible through alliances between for-profit and non-profit entities. Through the case study of alliances between forprofit companies and non-profit entities such as Grupo Vips-Fundacionhazlo Posible and Danone Foods-Grameen Bank, the reasons why these types of alliances are carried out are analyzed, as well as their implications and benefits, both for partner companies and for the communities in which they operate. The work analyzes these two cases and also performs a comparative analysis between the two in order to establish common behavior patterns in this type of alliance.
\end{abstract}

Keywords: Strategic alliances; Social responsibility; Shared value; Non-profit organizations.

Sumario. 1. Introducción. 2. Marco teórico. 3. Análisis de dos casos de alianzas estratégicas entre entidades lucrativas y no lucrativas: VIPS-Hazlo Posible y Danone-Grameen Bank. 4. Conclusiones. 5. Referencias bibliográficas.

Cómo citar. Talavera Avelino, C.; Sanchis Palacio, J.R. (2021) Ética y Responsabilidad Social en las alianzas estratégicas. El caso de las alianzas entre entidades lucrativas y no lucrativas. REVESCO. Revista de Estudios Cooperativos, vol. 137 , e73863. https://dx.doi.org/10.5209/reve.73863.

1 Universitat de Valencia, España.

Dirección de correo electrónico: carmen.talavera@uv.es.

2 Universitat de Valencia, España.

Dirección de correo electrónico: joan.r.sanchis@uv.es. 


\section{Introducción}

El entorno actual, caracterizado por una creciente rivalidad global, pero también por un progresivo aumento de concienciación sobre la importancia de aplicar políticas empresariales enfocadas al beneficio de la sociedad y el medioambiente, ha dado lugar, especialmente en las últimas décadas, a una re-concepción del mundo de los negocios. La ciudadanía demanda políticas empresariales que vayan más allá de la búsqueda del beneficio propio y se centren en la obtención de impactos positivos tanto sociales como medioambientales, hasta el punto en que la adopción de este tipo de comportamientos es ya necesaria para asegurar la competitividad de las organizaciones (Prado-Lorenzo et al., 2009; Mio, 2010; Harrison y Berman, 2016).

Esta nueva concepción ética y social del mundo de los negocios ha dado lugar a una emergente tendencia entre las empresas a llevar a cabo alianzas, con la intención de posibilitar la creación de valor compartido. Mediante la cooperación, las entidades pueden compartir recursos, acceder a nuevos bienes y capacidades, desarrollar conjuntamente nuevas tecnologías e incluso fortalecer sus posiciones competitivas a través de alianzas entre competidores. Las alianzas estratégicas permiten a las organizaciones un cierto nivel de competitividad que es muy difícil de alcanzar individualmente. Sin embargo, para que estos acuerdos proporcionen a los socios los beneficios esperados, gran parte de la literatura sobre la cooperación pone de manifiesto la importancia que los comportamientos éticos y las políticas de responsabilidad social tienen sobre ellos (Muñoz y Montoro, 2007; Pulgar y Pelekais, 2014; Thorne et al., 2017).

Con actitudes éticas basadas en la confianza mutua, las alianzas estratégicas podrán obtener los resultados esperados de su establecimiento. Para garantizar la efectividad de las medidas socialmente responsables, se pueden llevar a cabo alianzas con entidades que cuenten con competencias clave para su desarrollo. Así, se introduce la importancia de las alianzas entre empresas lucrativas y organizaciones sin ánimo de lucro, como base para el buen funcionamiento de las actividades de responsabilidad social empresarial y, por tanto, para la creación de valor tanto económico como social (Van der Voort et al., 2009; García et al., 2011; Carreras e Iglesias, 2012; Rodríguez et al., 2013; Abenoza et al., 2015). A través de este tipo de alianzas, no solo las empresas implicadas obtienen beneficios, sino también los diferentes actores sociales en los que éstas desarrollan su actividad. Por ello, el objetivo principal de este trabajo consiste en estudiar el vínculo entre los comportamientos éticos y socialmente responsables de las empresas a través de las alianzas estratégicas. Este objetivo se concreta en estudiar cómo se desenvuelven las alianzas entre empresas lucrativas y entidades no lucrativas, identificando las razones o motivos de las mismas y los beneficios que éstas producen, no solo en las empresas que cooperan, sino también para el conjunto de la sociedad. Con este propósito, se va a proceder a analizar en profundidad los siguientes aspectos: 1) los comportamientos éticos y socialmente responsables en las alianzas entre empresas; 2) las características de las alianzas entre empresas privadas y entidades no lucrativas como una forma de introducir la responsabilidad social en las empresas a través de las alianzas estratégicas; y 3) el impacto de las alianzas entre entidades lucrativas y no lucrativas para las organizaciones y la comunidad.

Para la consecución de estos objetivos, el trabajo se ha estructurado del siguiente modo. En primer lugar, se ha realizado un marco teórico, estructurado en torno a dos aspectos diferentes: la relación entre las alianzas estratégicas y los conceptos de ética y responsabilidad social en las organizaciones y el papel de las alianzas entre organizaciones con ánimo y sin ánimo de lucro. Tras ello, en segundo lugar, el trabajo incluye un estudio empírico, dentro del cual se describe la metodología cualitativa utilizada y el análisis de resultados obtenidos. Se realiza el análisis de dos casos de estudio de alianzas estratégicas entre entidades lucrativas y no lucrativas: VIPS-Fundación Hazlo Posible y Danone Foods-Grameen Bank. Para cada uno de ellos se realiza: una descripción de la historia de la alianza que incluirá el momento de su creación, su evolución y distintas etapas; se analizan los motivos del acuerdo y se estudia el impacto de estas alianzas sobre ambas empresas y sobre la sociedad; y se realiza una comparación de los resultados obtenidos para ambos casos para demostrar si existe un patrón común en las alianzas estratégicas entre organizaciones lucrativas y sin ánimo de lucro. El trabajo finaliza con un apartado de conclusiones.

\section{Marco teórico}

\subsection{Las alianzas estratégicas y su relación con la ética y la responsabilidad social}

Las características del contexto social y económico actual, en el que se observa un mayor dinamismo de la economía y una fuerte competitividad en mercados que son cada vez más globales, limitan la capacidad de las organizaciones para ser competitivas y hacen muy difícil que puedan progresar de forma individual. Con la intención de hacer frente a este complejo entorno y tener la posibilidad de ser más competitivas, en las últimas décadas, muchas entidades están llevando a cabo acuerdos de cooperación entre ellas (Muñoz y Montoro, 2007; Harrison y Berman, 2016, fortaleciendo así sus posiciones competitivas, asegurando su 
supervivencia y creando y manteniendo ventajas competitivas. Harrison y Berman (2016) destacan el papel de las alianzas en la adaptación a los ciclos económicos. Especial relevancia tiene la relación entre las empresas y sus proveedores, en lo que se refiere a la cadena de suministro bajo criterios de sostenibilidad (Canzaniello et al., 2017). El trabajo de Arenas y García (2007) destaca la relevancia de las alianzas a nivel internacional y el peso de la cultura y las relaciones de los socios en su éxito a nivel competitivo.

Sin embargo, para asegurar la durabilidad y especialmente la efectividad de este tipo de acuerdos de cooperación, es necesario que los socios, aparte de contribuir con recursos y capacidades, lo hagan también con atributos personales, como son la sinceridad, la integridad y la transparencia (Altamira, 2000), entre otros. De no ser así, tendrán lugar diferentes comportamientos que funcionarán como impedimento para la consecución de los objetivos establecidos con la alianza, como es el caso de los comportamientos oportunistas, que pueden dar lugar a la existencia de intereses distintos entre los socios, sin que haya claridad sobre ello, lo que dificultará la puesta en marcha de una estrategia en común, el incumplimiento de los compromisos por parte de alguno de los socios y la aparición de desconfianza a la hora de realizar un proyecto conjunto, por lo que no se delegará el poder o conocimiento necesario para desarrollar la actividad en común y ésta, por tanto, no podrá salir adelante (Fernández, 1999).

Además de los comportamientos oportunistas, existen otros inconvenientes a la hora de participar en un proceso de cooperación como son la pérdida de autonomía en la toma de decisiones y el incremento de los costes derivados de una mayor complejidad organizativa; aspectos que los socios deberán afrontar con paciencia, entereza y honradez o de lo contrario no podrán llegar a alcanzar los objetivos establecidos en la alianza (Lajara et al., 2003). De este modo, se introduce la importancia que los comportamientos éticos tienen en el funcionamiento de las alianzas estratégicas, tanto en el momento de su formación (NorheimHansen, 2013), con el objeto de aplicar acciones de responsabilidad social (Kopka et al., 2014; Pulgar y Pelekais, 2014) o para el caso de economías emergentes (Ma et al., 2016).

Las alianzas estratégicas son, por definición, acuerdos de cooperación a largo plazo en los que las empresas implicadas han de contribuir con sus propios bienes para la consecución de un objetivo común (Díaz, 2018). Sin embargo, la simple aportación por parte de los socios de una parte de sus recursos tangibles e intangibles no es suficiente para asegurar el éxito de las alianzas. Otro conjunto de factores relacionados con el comportamiento de las personas implicadas es necesario para garantizar la consecución de los objetivos esperados del acuerdo de cooperación (Michavila, 2011). Para que una alianza sea próspera, ésta, como punto de partida, ha de basarse en la confianza mutua entre los socios (Michavila, 2011) y tal y como menciona Renart Cava (1999), la aparición de conductas fraudulentas, oportunistas y carentes de ética por parte de los directivos y personal implicados en las alianzas destruye la confianza, convirtiéndose de este modo en una de las principales causas por las que éstas fracasan.

Con la intención de analizar la importancia e impacto que los comportamientos éticos tienen en las alianzas estratégicas, Renart Cava (1999) diferencia entre tres tipos de motivaciones: extrínseca, intrínseca y transcendental. Por un lado, el autor detalla que las personas pueden actuar movidas por una motivación de carácter extrínseca, esto es, por las recompensas materiales o económicas que esperan recibir por sus actuaciones. Por otro lado, define aquello que las personas esperan aprender o disfrutar de sus acciones como motivación intrínseca y por último, describe como motivación transcendente que las personas implicadas en una acción conozcan las consecuencias que sus decisiones sobre ésta tienen para ellos y para otras personas.

Para Pérez López (1993), la disposición motivacional de un directivo hacia la consecución de un objetivo común vendrá determinada por la sensibilidad que esta persona tiene para ser impulsada por cada una de estas tres definiciones de motivación. De esta manera, los comportamientos oportunistas surgen como consecuencia de una motivación extrínseca de los directivos, esto es, de su intención de conseguir ganar más dinero o vender más mediante la utilización de su socio. Sin embargo, como Renart Cava (1999) apunta, a largo plazo, esta conducta oportunista y falta de ética, ni siquiera dará los resultados necesarios para satisfacer las motivaciones extrínsecas de los directivos, puesto que la alianza acabará quebrándose. Por ello, para que la alianza estratégica brinde los beneficios esperados para ambos socios, es necesario que ésta se base en aspiraciones que van más allá de las motivaciones extrínsecas; debe sustentarse en decisiones tomadas con conocimiento de causa y con la intención de generar valor para todas las partes implicadas en el proceso a largo plazo, esto es, en la motivación transcendental y comportamientos éticos.

En esta línea de pensamiento, Browning et al. (1995), añaden que las alianzas más exitosas, no son aquellas en las que únicamente no tienen lugar comportamientos oportunistas y carentes de ética, sino aquellas en las que los compromisos adquiridos por los directivos y personas implicadas en el acuerdo de cooperación van incluso más allá de las responsabilidades exigidas por contrato. De este modo, los autores introducen el concepto de comunidad moral en las alianzas estratégicas. Estas comunidades son entendidas como vínculos basados en comportamientos éticos y valores morales, gracias a los cuales los socios otorgan a las alianzas aportaciones generosas y sinceras de sus conocimientos y recursos que exceden lo estipulado en el acuerdo de cooperación, fortaleciendo así el éxito de éstos.

Puede observarse, por tanto, como a lo largo de las últimas décadas diferentes estudios han centrado sus esfuerzos en explicar la relación que existe entre la ética y el éxito de las alianzas estratégicas en las 
organizaciones, desde diferentes enfoques como el del gobierno corporativo (Amran et al., 2014), la teoría de los stakeholders (Lai y Chen, 2014) y el enfoque territorial (Silvestri y Veltri, 2017). Asimismo, más recientemente, gran parte de la literatura sobre la cooperación empresarial ha enfocado sus trabajos en el impacto que las políticas de responsabilidad social, cada vez más desarrolladas en las entidades del siglo XXI, tienen en los acuerdos de cooperación: en la formación de la alianza (Norheim-Hansen, 2013), en el desarrollo de la alianza (Kopka et al., 2014; Pulgar y Pelekais, 2014) y en el control de la alianza (Ma et al., 2016).

La responsabilidad social corporativa puede ser definida como un modo de entender el mundo de los negocios, en el que las empresas son aliadas e impulsoras del desarrollo social y medioambiental, dando de esta manera, respuesta a las demandas de la sociedad actual (Kliksberg, 2009). Partiendo de esta definición, Rueda López (2016) considera que, por tanto, si el principio de responsabilidad social corporativa trata de abarcar una dimensión social y colectiva, la ética individualista en la que se basa el capitalismo no es apta para fundamentar la responsabilidad social; una ética basada en la cooperación y contribución mutua entre diferentes agentes parece de este modo necesaria.

Por su parte, Pulgar y Pelekais (2014) detallan la importancia de las alianzas estratégicas como medio para garantizar la efectividad de las políticas de responsabilidad social; a través de acuerdos de cooperación, aliados en diferentes o similares sectores pueden sumar sus fortalezas con la intención de resolver conjuntamente los desafíos que plantea la sociedad. En esta línea de pensamiento, Morales et al. (2011) mencionan la idea de que las medidas de responsabilidad social que llevan a cabo las organizaciones pueden ser más efectivas si se realizan cooperando con aquellas entidades que tengan las competencias clave para su implementación, esto es, a través de alianzas estratégicas con empresas de carácter social. De este modo, los autores, al igual que hacen Porter y Kramer (2011), introducen la importancia que tienen las alianzas estratégicas entre organizaciones capitalistas y entidades no lucrativas para la creación de valor compartido.

\subsection{Las alianzas estratégicas entre organizaciones lucrativas y no lucrativas}

Con la intención de analizar la importancia que tienen los acuerdos de cooperación entre entidades con ánimo de lucro y entidades no lucrativas, este apartado consistirá en un estudio sobre las razones de ser de las alianzas estratégicas entre estos dos modelos de organización y el impacto que estos acuerdos tienen para ambas empresas y la comunidad en la que se encuentran.

\section{Razón de ser de las alianzas estratégicas}

Si bien existe una gran variedad de autores que durante las últimas décadas han centrado sus investigaciones en la razón de ser de las alianzas estratégicas, el número de trabajos dirigidos al estudio de alianzas entre organizaciones lucrativas y no lucrativas es mucho más reciente y, por tanto, menor. Los trabajos incluidos dentro de este campo hacen referencia al estudio de este tipo de alianzas en sectores como la sanidad y la educación (Berger et al., 2004), el aprendizaje en las alianzas intersectoriales (Arya y Salk, 2006), las alianzas de empresas privadas con ONGs (Rivera-Santos y Rufin, 2010), alianzas intersectoriales enfocadas a webs corporativas (Shumate y O'Connor, 2010), alianzas enfocadas al uso del Balanced Scorecard (Hansen et al., 2010), alianzas enfocadas a la implementación de estrategias de marketing (Liu y Ko, 2011), alianzas intersectoriales enfocadas a la reputación (Im, 2011), alianzas enfocadas al desarrollo de estrategias (AlTabbaa, 2014) y alianzas enfocadas al papel mediador del altruismo (Rim et al., 2016). Sin embargo, en la literatura existente sobre el tema, se observa la importancia del concepto que distintos autores denominan como "creación de valor compartido" (Muthuri et al., 2009).

Por una parte, García et al., (2011) apuntan que las alianzas estratégicas entre entidades con ánimo de lucro y aquellas no lucrativas emergen como respuesta al cambio de necesidades e intereses que los diferentes stakeholders demandan a las organizaciones. Existen tres tipos distintos de perspectivas para llevar a cabo este tipo de alianzas, enfoques que han ido evolucionando a través de los años y que nombran, por orden cronológico, como: perspectiva de transacción, perspectiva de transición y perspectiva de transformación (García et al., 2011). En primer lugar, surge la perspectiva de transacción, basada en acciones filantrópicas que lleva a cabo la empresa para ayudar a la comunidad en un momento puntual. Debido a su carácter ocasional, estas no dan lugar a creación de valor ni para las entidades ni para la sociedad en su conjunto, sino que van destinadas a la mejora de un sector específico de la comunidad durante un período de tiempo determinado, en el que la empresa también se verá beneficiada, principalmente, a través de una mejora en su imagen. Los autores critican esta perspectiva puesto que las decisiones sobre qué acciones filantrópicas realizar para la comunidad, son tomadas en la empresa capitalista de forma unilateral, no en conjunto ni con la entidad no lucrativa ni con el resto de agentes de la sociedad.

En segundo lugar, y como respuesta a una demanda más exigente por parte de los grupos de interés, surge el enfoque de transición, como la construcción de puentes comunes entre la empresa y la sociedad a través de las alianzas. Para García et al. (2011), esta perspectiva implica diálogo continuo y una relación bilateral entra 
la empresa capitalista y la no lucrativa, relación en la que estas dos entidades tomarán las decisiones sobre qué políticas llevar a cabo para la mejora de la comunidad. Por tanto, este enfoque de las alianzas estratégicas entre empresas con ánimo de lucro y organizaciones no lucrativas, si bien incluye a la entidad no lucrativa en la toma de decisiones, deja fuera al resto de grupos de interés.

Por último, debido a la necesidad de incluir a los diferentes agentes sociales en la toma de decisiones sobre aquello que necesitan obtener de las alianzas entre entidades lucrativas y no lucrativas, aparece en tercer lugar el enfoque de transformación. García et al. (2011) definen esta perspectiva como la única que posibilita la creación de valor compartido tanto para la empresa como para la comunidad, puesto que en ella todos los agentes de la sociedad trabajan de forma multilateral para la consecución de objetivos comunes que permitan el aprendizaje y la creación de valor de todas las partes, aspecto que los autores consideran como la razón de ser de este tipo de alianzas; solo a través de la cooperación entre entidades lucrativas y no lucrativas, incluyendo en la toma de decisiones a todos y cada uno de los stakeholders de ambas organizaciones (lo que ellos consideran como una visión de "Responsabilidad Social Integral"), es posible la creación de valor compartido sostenible para todas y cada una de las partes.

Porter y Kramer (2011), al introducir el concepto de creación de valor compartido, ponen a su vez de manifiesto la idea de "empresa híbrida". Para los autores, operando de forma independiente, tanto las entidades lucrativas como las organizaciones sin ánimo de lucro están destinadas al fracaso, puesto que las primeras no tienen en cuenta las necesidades reales y demandas de todos sus grupos de interés y las segundas no garantizan la viabilidad económica de su actividad. Por ello, los autores datan la razón de ser de las alianzas estratégicas entre estos dos tipos de entidades en la necesidad de crear valor compartido con el fin de diluir las diferencias entre ambas y superar los obstáculos que cada una de ellas presentan para su propia supervivencia. A través de las alianzas estratégicas entre los dos tipos de organizaciones, las empresas son capaces de crear valor tanto económico como social, de modo que las entidades pasan de ser lucrativas o no lucrativas para convertirse en entidades de carácter híbrido (Porter y Kramer, 2011).

Puede concluirse, por tanto, que los estudios sobre el tema coinciden en que la motivación principal por la que las empresas lucrativas y las organizaciones sin ánimo de lucro llevan a cabo alianzas estratégicas es la creación de valor compartido. Asimismo, del mismo modo en que encontramos en la literatura diferentes trabajos destinados al estudio de este aspecto, también existen diversas investigaciones centradas en el impacto que esta creación de valor compartido tiene tanto para ambas organizaciones como para la comunidad.

\section{Impacto para los socios y para la comunidad de las alianzas estratégicas}

Con respecto al impacto que las alianzas estratégicas entre organizaciones lucrativas y no lucrativas representan para ambas organizaciones y para la comunidad, en la literatura sobre el tema se encuentran las siguientes investigaciones: American Express y Share Our Strength (organización contra el hambre) crearon una alianza en 1991 a través de la cual han desarrollado durante varios años una serie anual de eventos para recaudar fondos (Taste of the Nation) destinados a la investigación contra el cáncer (Berger et al., 2004); el desarrollo de procesos de aprendizaje en la introducción de prácticas de responsabilidad social mediante alianzas con entidades no lucrativas de inserción (Arya y Salk, 2006); alianzas B2N entre entidades no lucrativas y lucrativas que permiten limitar los comportamientos oportunistas de las empresas y mejorar su reputación (Rivera-Santos y Rufin, 2010); a partir de una muestra de 155 empresas norteamericanas del Fortune 500 y 695 ONGs, se detectan alianzas con éxito entre 11 empresas privadas y 59 entidades de inserción (Shumate y O'Connor, 2010); mediante el estudio del caso de las alianzas que mantiene Merck, Ltd. Tailandia (subsidiaria de una multinacional química y farmacéutica alemana) con entidades no lucrativas, se demuestran resultados tanto para la sociedad (para las comunidades locales) como para la propia empresa (reputación e imagen) ( Hansen et al., 2010); estudio mediante entrevistas semiestructuradas a gerentes de empresas lucrativas y entidades no lucrativas del Reino Unido demuestra la utilidad de estas alianzas para implementar estrategias de prácticas de responsabilidad social con impactos positivos en las comunidades locales (Liu y Ko, 2011); mediante el estudio del nacimiento de diferentes alianzas entre empresas con ánimo y sin ánimo de lucro se demuestra como las entidades no lucrativas se benefician de estas alianzas al obtener mayores fondos de financiación (Im, 2011); se analiza el papel activo de las entidades no lucrativas en la formulación de alianzas con empresas lucrativas y sus posibles beneficios (AlTabbaa, 2014); y por último, se demuestran efectos positivos en la reputación corporativa, sobre todo cuando existen motivos altruistas en las alianzas entre empresas privadas y entidades no lucrativas (Rim et al., 2016).

Tal y como se ha mencionado anteriormente en el trabajo, Porter y Kramer (2011) hablan de una difuminación en lo que a la definición histórica de cada tipo de entidad se refiere; a través de las alianzas estratégicas entre organizaciones lucrativas y no lucrativas, las empresas con ánimo de lucro pueden abarcar los aspectos de carácter social y medioambiental que predominan en las organizaciones no lucrativas y estas, a su vez, asegurarse la viabilidad económica necesaria para seguir operando. Gracias a los acuerdos de 
cooperación, ambas entidades pueden complementar sus deficiencias de modo que su supervivencia queda garantizada. Asimismo, como consecuencia de este tipo de acuerdos de cooperación, tiene lugar una creación de valor compartido fundamentado en la idea de que, a través de su actividad económica, las entidades son capaces de crear valor tanto económico como social (Porter y Kramer, 2011).

En la misma línea de pensamiento, estudios como el de Rodríguez et al. (2013), dotan de aportaciones más concretas en cuanto a la repercusión que este tipo de alianzas tienen para ambas organizaciones. El impacto positivo derivado de la cooperación entre entidades lucrativas y no lucrativas se observa a través de los siguientes factores: 1) primeramente, las empresas con ánimo de lucro se benefician a través de una mayor posibilidad de captación de nuevos socios gracias a una mejora de la imagen corporativa derivada de la alianza; 2) en segundo lugar, un aumento en la motivación de los trabajadores de ambas empresas, puesto que se necesitará tanto a nivel operativo como gerencial una mayor implicación de estos; y 3) por último, este tipo de alianzas, debido a la gran necesidad de cooperación y trabajo conjunto, permiten el intercambio de recursos tangibles e intangibles entre ambas organizaciones, aspecto que da lugar a un proceso de aprendizaje profundo que irá en aumento mientras dure la alianza y que permanecerá en cada una de las entidades en caso de que en un futuro el acuerdo de cooperación cesase. Además, los autores aluden a la importancia que este tipo de alianzas tienen para que entre las organizaciones lucrativas y sin ánimo de lucro, se establezca un marco común que posibilite el aprendizaje de las capacidades necesarias para competir en el entorno actual de un modo que dé respuesta a las necesidades de los diferentes agentes que componen la comunidad en la que se encuentran las entidades (Rodríguez et al., 2013).

Sobre este tema, Carreras e Iglesias (2012) señalan algunos de los beneficios mencionados en el apartado anterior y al mismo tiempo, añaden los siguientes aspectos. Con respecto a las ventajas reportadas de este tipo de alianzas para las empresas capitalistas señalan: 1) una mejora de sus procesos internos y la posibilidad de innovación en sus políticas de recursos humanos; 2) la retención de personal debido a un aumento de la motivación de éstos; 3) mayor visibilidad de los valores de la empresa y un incremento por parte de ésta en su concienciación sobre la realidad de la sociedad en la que se encuentra; 4) mejora en su posicionamiento de marca; y 5) una mayor facilidad a la hora de implementar políticas de responsabilidad social corporativa. En relación con los beneficios obtenidos por parte de la entidad no lucrativa, apuntan: 1) la captación de recursos tanto económicos como técnicos; 2) creación de nuevos servicios o productos gracias a la acción conjunta y el know how adquirido; 3) un aumento en sus canales de distribución; 4) acceso a muchos más destinatarios a través de las redes y contactos de la empresa capitalista; y 5) una mayor visibilidad ante la comunidad y ante otros socios potenciales.

Puede concluirse, por tanto, que existen diversos motivos por los que las organizaciones con ánimo de lucro y las no lucrativas llevan a cabo alianzas estratégicas, puesto que en los últimos años diversos autores han centrado sus investigaciones en los beneficios que este tipo de alianzas reportan a las empresas (Radrigán et al., 2012). Sin embargo, la importancia de estos acuerdos no reside únicamente en las ganancias ocasionadas para las organizaciones participantes de estos, puesto que en las alianzas entre entidades únicamente de carácter lucrativo, las empresas también encuentran gran cantidad de ventajas. Por ello, diversos autores han centrado sus investigaciones en la importancia que este tipo de acuerdos de cooperación tienen para la comunidad en la que se desarrollan (Van der Voort et al., 2009). Tal y como apuntan Abenoza et al. (2015), a través de este modelo de cooperación, existen tres tipos de impactos positivos para la comunidad. Por una parte, las entidades implicadas son capaces de resolver un problema social, gracias a la puesta en común de sus divergentes recursos, por lo que consiguen llevar a cabo proyectos comunitarios de un modo mucho más eficiente de lo que podrían hacerlo en el caso de operar por separado.

Por otro lado, los autores mencionan que, a través de este tipo de alianzas, no solo pueden resolverse problemas de carácter comunitario, sino que también tiene lugar la generación de innovación social (Abenoza et al., 2015). Como consecuencia de la cooperación entre empresa lucrativa y no lucrativa, se pueden desarrollar nuevos productos, servicios y/o tecnologías que tengan un importante impacto social, esto es, esta clase de alianzas dan lugar a la creación de ofertas innovadoras destinadas a la resolución de los problemas sociales mencionados anteriormente. La entidad no lucrativa detecta el reto social o medioambiental y aporta sus conocimientos sobre ello, mientras que la empresa con ánimo de lucro aportará su experiencia profesional en cuanto al desarrollo de productos o servicios se refiere.

Por último, el tercer beneficio comunitario que Abenoza et al. (2015) indican sobre este tipo de alianzas, tiene que ver con los cambios tanto locales como globales que estas promueven. En este caso, se refieren a este tipo de acuerdos de cooperación como alianzas estratégicas llevadas a cabo con la intención de promover prácticas éticas, a las que denominan global action network, puesto que las describen como coaliciones creadas para promover cambios no solo locales, sino también a nivel global. Estos acuerdos se basan en el aprendizaje compartido entre las empresas y en el establecimiento de redes innovadoras surgidas a partir del intercambio de tecnología entre ambas entidades; tienen un carácter de transformación social y, por tanto, no se restringen a la comunidad local en la que se encuentran, sino que pretenden tener un impacto positivo integral a través de la innovación. En esta línea de pensamiento, Callejas y Salazar (2012) indican que el intercambio de conocimientos y tecnología que tiene lugar entre las organizaciones con ánimo de 
lucro y las no lucrativas, deja una huella comunitaria tanto local como global, que puede observarse en ambos niveles, el social y el medioambiental. Este tipo de alianzas permiten, a través de la innovación, el desarrollo e implementación de nuevos modelos de producción y distribución de productos y servicios destinados al bien común que, a su vez, son amables con el medioambiente y que no han de porqué restringirse exclusivamente a la localidad en la que las organizaciones se encuentran.

La transcendencia, por tanto, de las alianzas estratégicas entre organizaciones lucrativas y no lucrativas reside en el impacto que éstas tienen en la comunidad; únicamente a través de este modelo de cooperación es posible la creación de valor sostenible, tanto económico como social, puesto que este tipo de alianzas estratégicas posibilitan el desarrollo social a través del desarrollo económico y viceversa (Romero y Oliva, 2012).

\section{Análisis de dos casos de alianzas estratégicas entre entidades lucrativas y no lucrativas: VIPS-Hazlo Posible y Danone-Grameen Bank}

\subsection{Metodología}

Se ha elegido la metodología cualitativa ya que, como afirma Yin (2013), a través del análisis de casos, dado el carácter crítico y peculiar de cada uno de ellos y el objeto de estudio único que comprenden, se pueden realizar investigaciones y extraer conclusiones que no serían posibles de otra manera. Asimismo, el estudio de casos múltiple (trabajos en los que se utilizan varios casos a la vez), permite estudiar y comparar ejemplos y hechos de manera que finalmente puede llegar a describirse una realidad (Yin, 2013). En la misma línea de pensamiento, autores como Rule y John (2015) afirman que es posible comprobar y afirmar teorías a través del estudio de casos. Su estudio indica que, del mismo modo en que las metodologías cuantitativas permiten el establecimiento de hipótesis, testarlas en la práctica y su comprobación a través de operaciones numéricas, las metodologías cualitativas permiten testar y comprobar teorías a través del estudio de casos, por medio de deducciones que se mueven de lo general a lo específico.

Para los autores, el estudio de varios casos proporciona la base para la generalización, esto es, indican que, si bien dicho estudio de casos individuales podría dar lugar a generalizaciones difusas, mediante el estudio de casos múltiples pueden comprobarse relaciones entre factores; relaciones que al aplicarse a otros contextos proporcionan un mayor alcance para la generalización. Si a través del estudio de múltiples casos se observa que un mismo fenómeno tiene lugar en varios contextos diferentes, esto puede ser un indicador que revele una tendencia más amplia, es decir, el fenómeno puede estar revelando una tendencia que puede ser atribuida a una escala mayor (Rule y John, 2015). Esta generalización es descrita por Yin (2002) como analytic generalization (generalización analítica), proceso que, según indica el autor, tiene lugar cuando una teoría puede utilizarse como base o plantilla con la que comparar los resultados empíricos obtenidos con el estudio de los casos; si mediante el estudio de múltiples casos, los resultados obtenidos apoyan la misma teoría, esta puede generalizarse y extrapolarse a otros casos.

Puesto que el objetivo final de este trabajo es demostrar las teorías expuestas en el marco teórico (las razones de ser y beneficios que las alianzas estratégicas entre entidades lucrativas y no lucrativas reportan tanto para las organizaciones envueltas en los acuerdos de cooperación como para la comunidad en la que se encuentran), se va a realizar un estudio empírico constituido por el análisis y comparación de dos casos de estudio; casos seleccionados en base a la notoriedad de las empresas participantes y la cantidad de información publicada con respecto a los acuerdos de cooperación llevados a cabo entre ellas. El estudio se va a dividir en tres apartados: estudio individual de la alianza entre Grupo Vips y Fundación Hazlo Posible, estudio individual de la alianza entre Danone Foods y Grameen Bank y análisis comparativo de ambos casos, con el propósito de discernir si existe o no un patrón común entre los acuerdos.

\subsection{Estudio del caso de la alianza Grupo VIPS-Fundación Hazlo Posible ${ }^{3}$}

El estudio de la alianza entre Grupo VIPS y Fundación Hazlo Posible se va a realizar en base a tres aspectos: 1) la historia de la alianza, que incluirá sus diferentes etapas y evolución; 2) las razones de ser y motivos de la alianza; y 3) sus implicaciones y beneficio, tanto para las entidades involucradas como para la comunidad.

\section{Historia de la alianza}

Grupo Vips es una entidad familiar creada en Madrid en 1969, momento en el que su fundador, Plácido Arango, decidió abrir un primer establecimiento bajo el nombre Vips. Si bien en sus inicios, la compañía 
contaba únicamente con una cadena de restaurantes, actualmente es un grupo formado por 6 grandes cadenas (Vips, Ginos, The Wok, Restaurantes Tío Pepe, T.G.I Friday's y Starbucks Coffee) y 10 restaurantes (El Bodegón, Teatriz, Bice, Iroco, Lucca, Tattaglia, Rugantino, Paparazzi, Manzoni y Root), con más de 350 establecimientos y con una plantilla superior a los 10.000 empleados.

Fundación Hazlo Posible es una entidad privada sin ánimo de lucro creada en 1999 por José Martín y Catalina Parra, con el fin de estimular de un modo innovador la implicación de la sociedad en las causas solidarias y sociales, utilizando para ello las tecnologías de la información y comunicación. Con esta intención, crearon un portal de internet llamado Canalsolidario.org, destinado a servir como canal de unión entre las actividades de la ONG y la sociedad, siendo este el primer portal español que contaba con una gran cantidad de información sobre las ONG. Actualmente, Canalsolidario.org es un periódico digital que se especializa en información de carácter social, mientras que la Fundación centra sus operaciones en tres líneas de acción: la sensibilización y comunicación, el voluntariado y la gestión del conocimiento. Cuenta con una plantilla de 17 personas y un censo de 500 voluntarios que colaboran con la organización rigiéndose por los valores de compromiso, diversidad, energía y creatividad.

La colaboración entre ambas empresas comienza en 1999 tras un encuentro fortuito que tuvo lugar en la primera feria de Internet en Madrid entre Maite Arango, vicepresidenta del Consejo de Grupo Vips, y Elena Acín, directora de la Fundación Hazlo Posible por aquel momento. Elena Acín presentaba en la feria por primera vez el portal Canalsolidario.org en busca de financiación privada y a su vez, Maite Arango, buscaba un proyecto social en el que Grupo Vips pudiera comprometerse. Tras la realización de posteriores reuniones, en el año 2000, ambas organizaciones acuerdan colaborar conjuntamente en el portal Canalsolidario.org, el cual pasa a denominarse Hacesfalta.org y en el que Grupo Vips realizó una inversión inicial de 106.000 euros. A partir de este momento comienza la alianza estratégica, coalición en cuya evolución se observan tres etapas distintas.

La primera etapa de formación se centra en construir las bases de la relación de colaboración bajo una atmósfera de entendimiento y compatibilidad. Ambas organizaciones coincidían en su intención de dar visibilidad a un nuevo tipo de voluntariado en España, el voluntariado virtual, y con este propósito, mediante reuniones bimensuales de carácter informal, unieron sus conocimientos: los profesionales de Grupo Vips aportaron su experiencia de atención al cliente y desde la Fundación Hazlo Posible sus habilidades en consultoría, por lo que el lanzamiento del portal tuvo un gran éxito a principios de los 2000. Sin embargo, con la intención de garantizar el cumplimiento de los acuerdos, es en este período cuando se empiezan a establecer mecanismos formales de gobernanza a través de la firma de convenios anuales y la presentación de informes mensuales para el seguimiento del desarrollo del proyecto.

La segunda etapa de la alianza, la fase de operación, la cual se caracteriza por un reforzamiento de los acuerdos de colaboración entre ambas organizaciones, comienza en 2003 cuando Fundación Hazlo Posible crea un programa de voluntariado corporativo como extensión de Hacesfalta.org, en el que pudieran participar diferentes empresas, y que lanza a inicios de 2004. Para gestionar este programa, Grupo Vips crea su nuevo departamento de responsabilidad social corporativa y las relaciones pasaron a ser tanto de carácter formal como informal, gracias al fuerte nivel de compromiso e interacciones entre ambas; factores que propiciaron la aparición de la confianza. La confianza ha funcionado como un mecanismo de gobernanza esencial para el desarrollo de esta alianza estratégica a largo plazo, aspecto que como se ha citado anteriormente en el trabajo, Michavila (2011) considera como punto de partida para que una alianza sea próspera.

La tercera etapa de la alianza estratégica, la fase de desarrollo comienza en 2005 con la creación del proyecto Brazos Abiertos, como parte del Portal de Voluntariado Corporativo, y destinado a integrar a los nuevos empleados en las empresas y a los emigrantes llegados a España en la sociedad. Debido al crecimiento de los diferentes proyectos llevados a cabo, fueron necesarios cambios organizativos que derivaron en una renovación en los puestos directivos de ambas organizaciones y en la incorporación de diversas personas a la plantilla de trabajadores.

En los próximos tres años, debido al fuerte crecimiento y con intención de prolongar esta tendencia y hacer frente a la crisis económica de 2008, Fundación Hazlo Posible se ve en la necesidad de buscar nuevas fuentes de financiación. Por ello, en 2010, firma un acuerdo con Grupo Vips a 3 años en el que se especifica que el presupuesto aportado por el Grupo aumentará en ese año con respecto a años anteriores, para reducirse de forma progresiva en los dos años siguientes. A cambio de esta reducción, la Fundación buscaría nuevas fuentes de financiación a través de otros patrocinios y contratos publicitarios, siempre que éstos fuesen aprobados por la dirección de Grupo Vips. De esta manera, en 2010, se afianza la alianza estratégica entre ambas organizaciones, gracias a la abertura de la Fundación a nuevas inversiones. Gracias a esta abertura, en la actualidad las entidades están trabajando conjuntamente en el proyecto Hacesfalta.org y en un nuevo programa incorporado en marzo de 2019, el cual forma parte del Portal de Voluntariado corporativo, bajo el nombre Red Talento Que Impacta (Grupo Vips, 2020b). Este nuevo proyecto está destinado a promover las relaciones estratégicas entre organizaciones lucrativas y no lucrativas, a partir de espacios de encuentro, con 
la intención de impulsar la transferencia de conocimiento y aprendizaje entre ambos tipos de empresas, con el propósito final de potenciar y fortalecer la creación de valor social en la comunidad (Hazlo Posible, 2019).

\section{Razones de ser de la alianza}

Como se ha mencionado en el apartado anterior, la alianza estratégica entre Grupo Vips y Fundación Hazlo Posible nace en 1999 como consecuencia de un encuentro casual entres dos miembros del equipo directivo de ambas organizaciones. Por un lado, Acín, directora de la Fundación Hazlo Posible, consciente de que formaba parte de una ONG nueva y de la dificultad que ello supone para acceder a financiación pública, buscaba una fuente de ingresos privada para poder crecer de un modo más rápido de lo que el crecimiento interno lo permite. Por otro lado, Arango, vicepresidenta de Grupo Vips, explorando nuevas alternativas de crecimiento que respetasen la misión y valores de la organización (consideración de las personas y su diversidad, trabajo en equipo, satisfacción de los clientes y contribución positiva a la sociedad), buscaba un proyecto de carácter social e innovador en el que la empresa se pudiera emprender.

Por tanto, puede observarse que el principal motivo o razón de ser del acuerdo de cooperación entre Grupo Vips y la Fundación Hazlo Posible es la posibilidad de acceder a recursos necesarios de los que disponen los socios, para desarrollar la actividad empresarial de manera óptima. Fundación Hazlo Posible buscaba recursos financieros mientras que Grupo Vips buscaba conocimientos y experiencia en aspectos sociales. De esta manera, tal y como se ha mencionado en el marco teórico como una de las razones de ser de los acuerdos de cooperación, la alianza se lleva a cabo para alcanzar capacidades y comportamientos complementarios a través de la cooperación (Contractor y Lorange, 1988). Gracias a la alianza, la Fundación Hazlo Posible consigue la financiación necesaria que le permite crecer y desarrollar su actividad y a su vez, Grupo Vips, se embarca en un proyecto que le posibilita adentrarse en un nuevo modelo de negocio acorde con su estrategia y valores empresariales.

Asimismo, Arenas y García (2007) señalan que la creación y mantenimiento de ventajas competitivas es también uno de los principales motivos por los que se establecen alianzas estratégicas. Relacionado con esta afirmación, Planellas y Urriolagoitia (2011) afirman que el acuerdo de cooperación ha proporcionado una ventaja competitiva sostenible tanto a Grupo Vips como a la Fundación Hazlo Posible; debido a los conocimientos y experiencias compartidos a lo largo de las diferentes etapas, la coalición se ha convertido en una fuente de capacidad distintiva que genera beneficios significativos para ambas organizaciones ${ }^{4}$.

Si bien existen diferentes razones de ser por las que se llevó a cabo la alianza, ésta no hubiera sido posible de no ser porque ambas organizaciones compartían un objetivo común. Tal y como apuntan Gaona et al., (2019), pueden existir diferentes razones por las que establecer un acuerdo de cooperación, pero hay una coexistente en todos ellos: la cooperación estratégica ha de tener como base la intención final por parte de los socios de lograr un objetivo común, de manera que ambos estén comprometidos a la consecución de este. La posibilidad de acceder a recursos de los que disponen los socios, desenvolver conjuntamente la actividad empresarial de manera óptima, desarrollar nuevos productos o servicios o la creación y mantenimiento de ventajas competitivas sostenibles son motivos de ser de la alianza Grupo Vips-Fundación Hazlo Posible, pero a su vez, también el objetivo común que comparten ambas entidades, siendo este la base de su creación.

\section{Implicaciones y beneficios de la alianza}

Con respecto al impacto que la alianza estratégica ha reportado tanto para las dos empresas como para la sociedad, encontramos las siguientes implicaciones. En relación con el apartado anterior sobre el importante papel que la combinación de recursos tangibles e intangibles ha significado en el acuerdo de cooperación entre Grupo Vips y la Fundación Hazlo Posible, cabe destacar que esta transferencia de know how ha dado lugar a la generación de una sinergia única, valiosa e inimitable. Como consecuencia de reiteradas interacciones entre ambas organizaciones aportando y combinando recursos y capacidades complementarios, se pudieron implementar los diferentes proyectos citados alrededor de este trabajo que se han convertido en una fuente de beneficios para ambas entidades y la comunidad. Por tanto, puede observarse como gracias a la adquisición de nuevos recursos y capacidades a través de la cooperación, tiene lugar el surgimiento de un conocimiento conjunto único que posibilita la creación de una ventaja competitiva, la cual será sostenible como consecuencia de su carácter singular. Por ello, Arenas y García (2007) apuntan que este know how y experiencia conjunta emanados de la alianza, son uno de los principales beneficios reportados para las organizaciones de los acuerdos de cooperación.

De igual modo, en lo que respecta a los beneficios para las dos organizaciones y con respecto a la teoría mencionada por Rodríguez et al., (2013), quienes afirman que las organizaciones se benefician a través de una mayor posibilidad de capacitación de nuevos socios gracias a una mejora de la imagen corporativa 
derivada de la alianza, cabe señalar que, en menos de un año tras establecer el acuerdo, DKV, ONO y Bankinter se añadieron al proyecto. Asimismo, en 2007, Accenture, Mapfre, Cajamar y BBVA se incorporaron al Portal de Voluntariado, aumentando así significativamente el número de socios de la alianza. En la actualidad, la Fundación Hazlo Posible cuenta con 53 socios y forma parte de 10 redes de carácter social (Hazlo Posible, 2020). Este aumento en el número de socios se encuentra estrechamente relacionado con una mejora de la reputación empresarial, especialmente para la entidad lucrativa Grupo Vips. Se observa que los valores de cada una de las organizaciones han constituido una cultura basada en la diversidad, innovación, trabajo en equipo y eficiencia. Esta cultura ha sido gratamente percibida por los diferentes stakeholders de las entidades, contribuyendo así a un aumento de la reputación empresarial.

En línea con el pensamiento de Rodríguez et al., (2013), cuyo estudio menciona la relación positiva entre la reputación corporativa y la cooperación entre organizaciones con ánimo y sin ánimo de lucro, desde el establecimiento de la alianza, Grupo Vips es considerada como una de las empresas más reputadas del sector. Organizaciones como MERCO (Monitor Español de Reputación Corporativa) y la Fundación Empresa y Sociedad han otorgado al Grupo Vips la consideración de empresa mejor percibida del sector debido a su acción social. Asimismo, ha recibido varios premios por sus acciones de responsabilidad social corporativa, como el otorgado por la Federación Española de Hostelería (FEHR) en 2009 o el galardón de la Fundación Bip Bip en ese mismo año. Cabe destacar que tanto Grupo Vips como la Fundación Hazlo Posible son, desde la instauración de la alianza, invitados a foros académicos y cursos para hablar sobre las claves de su éxito, además de ser citados por ello en diversas publicaciones y revistas académicas. Todo ello, le ha permitido al Grupo Vips crecer de una manera exponencial en los últimos años, pues de una plantilla de 2.800 empleados en 1999, ha pasado a alcanzar en la actualidad los 9.300 trabajadores, repartidos entre los 400 establecimientos y 6 cadenas de la compañía (Grupo Vips, 2020a).

Cabe destacar en lo relacionado a los empleados del grupo, el impacto positivo que la coalición ha tenido sobre éstos. Carreras e Iglesias (2012) indican que las alianzas estratégicas entre entidades lucrativas y no lucrativas suponen un aumento de la retención de la plantilla de trabajadores debido a un incremento de la motivación de éstos. Relacionado con esta afirmación, se observa en el caso analizado que, gracias a la creación del Portal de Voluntariado destinado a integrar en diferentes empresas a los nuevos y existentes trabajadores, las compañías constataron que éste constituía un motivo de refuerzo de los valores de solidaridad y responsabilidad empresarial. Asimismo, el portal reforzaba la comunicación y relaciones interpersonales entre diferentes divisiones de las entidades, aumentando el orgullo de pertenencia y contribuyendo de esta manera a una mayor motivación y retención del personal, por lo que se produjo una disminución del índice de rotación posterior a la alianza.

Con respecto al beneficio reportado a diferentes grupos de la comunidad, en primer lugar y estrechamente relacionado con la afirmación anterior, cabe destacar que, gracias a la creación del Portal de Voluntariado, este acuerdo de cooperación ha contribuido a potenciar la cultura del voluntariado en España, puesto que desde su implantación en 2004 cientos de empresas han participado activamente en iniciativas solidarias. En segundo lugar, la alianza también ha contribuido al desarrollo tecnológico. Si bien con anterioridad al establecimiento de la alianza, la Fundación Hazlo Posible ya contaba con el portal de internet Canaldolidario.org, fue gracias a la financiación aportada por Grupo Vips que éste pudo desarrollarse y llegar a tener la repercusión social que tuvo, junto con posteriores iniciativas creadas de manera conjunta. Por tanto, este hecho confirma la teoría de Abenoza et al. (2015), que afirman que como consecuencia de este tipo de acuerdos de cooperación se pueden desarrollar nuevos productos y servicios innovadores destinados a la resolución de problemas sociales, como es el caso de los portales de Internet perfeccionados y creados durante las diferentes etapas de la alianza.

Por último, cabe destacar la creación de una ventaja competitiva sostenible, aspecto ya introducido en el apartado anterior. Las sinergias originadas como consecuencia de la transferencia y complementariedad de recursos y conocimientos han propiciado la aparición de sinergias en los departamentos de recursos humanos e innovación, sinergias de carácter único y sostenible gracias a la confianza establecida entre ambas organizaciones. Esta ventaja competitiva sostenible se basa, como afirman Porter y Kramer (2011), en la creación de valor compartido: ambas organizaciones han conseguido asegurar su viabilidad económica y supervivencia a través de la creación de valor tanto económico como social, gracias a su trabajo conjunto; Grupo Vips aportando financiación y redes de contactos y la Fundación Hazlo Posible colaborando con su conocimiento sobre aspectos de carácter social.

\subsection{Estudio del caso de la alianza Danone Foods-Grameen Bank ${ }^{5}$}

Al igual que en el caso anterior, se va a analizar en base a tres aspectos: 1) la historia de la alianza, que incluirá sus diferentes etapas y evolución; 2) las razones de ser y motivos de la alianza; y 3) sus implicaciones y beneficio, tanto para las entidades involucradas como para la comunidad. 


\section{Historia de la alianza}

Danone-Foods es un grupo de alimentación fundado por Isaac Carasso en 1919 con el propósito de mejorar la salud de las personas a través del progreso en el proceso de fermentación. Si bien la primera fábrica artesanal fue establecida en Barcelona, en el año 1929, la compañía se trasladó a Francia, donde se creó la primera fábrica destinada a la producción de yogures. En la actualidad, la corporación cuenta con cuatro líneas de negocio: productos lácteos, aguas, nutrición infantil y nutrición médica; disponiendo de 30 marcas distintas en las que opera en más de 130 países repartidos en los 5 continentes (Danone, 2020).

Por su parte, el Banco Grameen es una entidad creada en 1940 por Muhammad Yunus en Bangladesh con la intención de ofrecer microcréditos a la población más empobrecida sin necesidad de garantías. La idea de Yunus era otorgar capacidad de financiación a las personas más pobres, en términos y condiciones que pudiesen afrontar de forma razonable y de este modo realizar pequeñas actividades que contribuyan al desarrollo de la comunidad. Actualmente, el Banco Grameen cuenta con 2.568 sucursales, una plantilla de 21.751 trabajadores y una base de clientes de alrededor de nueve millones de prestatarios en más de 80.000 aldeas. Cabe destacar que los métodos empleados por Grameen se aplican en proyectos en 58 países, entre ellos Estados Unidos, Canadá, Países Bajos, Francia y Noruega (Grameen, 2020).

La alianza estratégica entre ambas organizaciones se remonta al año 2005, momento en el que Yunus visitó la ciudad de París y recibió una invitación por parte de Franck Riboud, CEO del grupo Danone, para reunirse. En esta reunión, el fundador de Grameen le propuso a Riboud la creación de un acuerdo de colaboración entre ambas entidades para tratar de abordar de forma conjunta el problema de la desnutrición infantil en Bangladesh, especialmente en las zonas rurales del país. Con este propósito, en 2006, mediante una joint venture se crea Grameen Danone Foods, bajo el modelo de empresa social. Este modelo de negocio tiene como centro de sus operaciones la resolución de problemas sociales y medioambientales, por lo que los beneficios obtenidos son reinvertidos en la organización, de modo que se crea un círculo de retroalimentación que genera crecimiento contribuyendo al desarrollo. Esta empresa social, si bien se basa en la consecución o mejora de una acción comunitaria, debe ser rentable, de tal manera que las ganancias puedan reinvertirse para asistir a un proceso continuo de avance y progreso (Barrera, 2007).

De esta manera, la joint venture de carácter social Grameen Danone Foods comienza a fabricar un nuevo yogurt bajo el nombre de Shoktidoi, que significa "yogurt fuerte". Este producto, elaborado con nutrientes esenciales para el desarrollo, está específicamente diseñado para suplir las deficiencias nutricionales de los niños y niñas de Bangladesh a un precio de venta de 8 céntimos de dólar, siendo alcanzable para la población más empobrecida (Danone Communities, 2020a). En 2006, se establece en la ciudad de Bogra la primera planta destinada a la producción de este nuevo Shoktidoi; una fábrica 50 veces más pequeña que las plantas estándar del grupo Danone, la cual abastece a una población rural distribuida en un perímetro de 30 kilómetros alrededor de la fábrica (Danone Communities, 2020b). Desde la institución de esta planta, se han invertido alrededor de 700 mil dólares en la región y más de 7 millones de personas se han visto beneficiadas a través del acceso a productos de salud, higiene, nutrición y empleo (Fundación Seres, 2020).

\section{Razones de ser de la alianza}

Como puede observarse, Grameen Danone Foods se crea con la intención de dar acceso diario a alimentos nutritivos a la población con menos ingresos de Bangladesh e implicar a la comunidad en la producción y distribución de los productos alimentarios; todo ello con el objetivo último de mejorar las condiciones económicas de la población más empobrecida del país, implicándolos en el proceso.

Por una parte, como empresa social, Grameen Danone Foods nace con el propósito de crear valor social, anteponiendo de esta manera el beneficio colectivo al económico. Por ello, las empresas sociales tienen por objetivo asistir a las personas en riesgo de exclusión o que se encuentran en una situación que les supone un estado de indefensión. Como se ha mencionado en el apartado anterior y en línea con los fundamentos de la empresa social, Grameen Danone Foods se crea para producir y distribuir yogures con un alto contenido nutricional a la población de Bangladesh más empobrecida. De esta manera, citando la teoría expuesta por Contractor y Lorange (1988), puede observarse que la alianza se establece con el propósito de alcanzar capacidades y comportamientos complementarios a través de la cooperación y el acceso más rápido a nuevos mercados. Por un lado, Grameen aporta sus conocimientos sobre el mercado y población de Bangladesh, así como su experiencia en cuestiones de carácter social y, por otro lado, Danone contribuye con su know how sobre cómo producir yogures nutritivos, asegurándose al mismo tiempo, la entrada al mercado de Bangladesh.

Por otra parte, cabe destacar, que en este modelo de empresa los colectivos en riesgo de exclusión no son únicamente considerados como el cliente de la organización, sino que son parte de ella, convirtiéndose así en una parte activa de la empresa mediante la propiedad de esta o la inserción de las personas en alguno de los procesos de la cadena de valor, como puede ser desde el cometido de proveedores, productores o distribuidores. En el caso de Grameen Danone Foods, ésta se establece no únicamente con el objetivo de 
ofrecer productos alimenticios al sector de la población que más lo necesita, sino de asegurarse la inserción de éstos en los procesos de producción y distribución de los yogures. De este modo, el beneficio social generado va más allá de una mejora en la alimentación de quienes más lo necesitan, puesto que se crea empleo, lo que a su vez genera valor económico; beneficio que, al reinvertirse en la organización, permite seguir operando y aumentar, por tanto, el valor social. De esta manera, con el establecimiento de Grameen Danone Foods, las entidades consiguen crear una ventaja competitiva sustentada en la creación tanto de valor social como económico, de modo que se aseguran la supervivencia de la organización (Arenas y García, 2007).

\section{Implicaciones y beneficios de la alianza}

Con respecto al impacto que la alianza estratégica ha reportado para las organizaciones y la sociedad, encontramos las siguientes implicaciones. En primer lugar, en relación con los beneficios aportados a la empresa con ánimo de lucro (Danone) y haciendo referencia a la teoría expuesta anteriormente en el trabajo por Carreras e Iglesias (2012), como consecuencia de la alianza, Danone se ha beneficiado de una mayor facilidad a la hora de implementar políticas de responsabilidad social corporativa. Asimismo, y relacionado con este aspecto, tal y como apuntaban Rodríguez et al. (2013), Danone Foods ha experimentado un aumento en su número de socios gracias a una mejora de su imagen corporativa derivada del acuerdo de cooperación. El establecimiento de la empresa social Grameen-Danone Foods en 2006, se convirtió en el proyecto fundacional de Danone Communities, red de negocios sociales destinada a la asistencia de emprendedores locales con financiación y formación (Corporate Danone, 2020a). En la actualidad, Danone Communities cuenta con once negocios sociales, de los cuales forman parte diferentes entidades con ánimo de lucro (Danone Communities, 2020a).

Cabe destacar, citando una vez más a Carreas e Iglesias (2012), que la creación de Danone Communities, ha supuesto una mejora significativa del posicionamiento de marca del grupo. Según el índice Reptrak, indicador de reputación corporativa emitido por el Reputation Institute (Reptrak, 2020), entre los años 2010 y 2014, Grupo Danone ocupó las primeras posiciones del ranking; siendo reconocida en el año 2015 como la empresa con mejor reputación del sector de alimentación y bebidas (Corporate Danone, 2020b).

En segundo lugar, con lo que respecta a las implicaciones para la entidad sin ánimo de lucro (Grameen Bank), del mismo modo que ocurre con el Grupo Danone, se observa que la alianza estratégica también ha supuesto una mayor visibilidad del Banco Grameen ante la comunidad y ante otros socios potenciales (Carreras e Iglesias, 2012). Desde la creación de Grameen-Danone Foods en 2006, la entidad no lucrativa ha sido partícipe en la creación de siete organizaciones de carácter social, (Grameen Foundation, 2020); hecho que tal y como citaban Carreas e Iglesias (2012), le ha permitido acceder a muchos más destinatarios a través de la creación de nuevos servicios o productos gracias a la acción conjunta y el know how adquirido. Asimismo, citando el estudio mencionado en el marco teórico de Rodríguez et al. (2013), gracias al acuerdo de cooperación, se ha producido el establecimiento de un marco común que ha dado lugar al aprendizaje de las capacidades necesarias para competir en el entorno actual de un modo que dé respuesta a las necesidades de los diferentes agentes que componen la comunidad en la que se encuentran las entidades; aspecto que puede observarse en los beneficios reportados a la sociedad.

Por tanto, en tercer lugar, con relación al impacto social y comunitario de la alianza, encontramos los siguientes aspectos tanto en la esfera medioambiental, como en la económica y social. Primeramente, en relación al beneficio e impacto positivo en el medio ambiente, tal y como mencionan Fisac et al. (2011) en su estudio realizado sobre este acuerdo de cooperación, la intención de Danone Foods y Grameen Bank fue desde los inicios de la alianza reducir el impacto medioambiental de sus operaciones conjuntas; motivo por el que la fábrica situada en la ciudad de Bogra funciona con energía solar destinada a calentar el agua empleada en las calderas principales y en la limpieza de las instalaciones.

Por otro lado, como parte de la repercusión económica que la empresa social ha supuesto en Bangladesh, cabe destacar que ésta se ha convertido en una importante fuente de creación de ingresos para la región de Bogra y sus alrededores. Por una parte, la leche utilizada para la fabricación de los yogures proviene de 475 agricultores situados en un área de 30 kilómetros alrededor de la planta y por otro lado, de la distribución de éstos se encargan 250 mujeres microempresarias de la zona, quienes se ocupan de venderlos puerta a puerta. Asimismo, cabe destacar que 30 personas locales trabajan en la elaboración de los yogures, siendo un total de 1.600 empleados los que componen la plantilla de Grameen Danone Foods. De este modo, se pone de manifiesto como diferentes agentes de la comunidad participan en los procesos de elaboración y distribución de los productos alimenticios; por lo que no solo se consigue mejorar la alimentación de los niños, sino que tiene lugar la creación de un tejido empresarial que ofrece oportunidades de empleo y, por tanto, de progreso, a diferentes sectores de la comunidad.

De esta manera, Grameen Bank y Danone Foods, con su alianza, consiguen dar lugar a la creación de valor compartido; valor fundamentado en la idea de que a través de su actividad económica (en este caso la fabricación de yogures), las organizaciones implicadas son capaces de crear tanto valor económico como 
social (Porter y Kramer, 2011). Asimismo, con el establecimiento de Grameen-Danone Foods, las organizaciones consiguen crear una empresa social, diluyendo de esta manera la idea o definición histórica que se tiene de cada tipo de entidad; ambas crean una organización de carácter híbrido, en la que, citando una vez más el estudio realizado por Porter y Kramer (2011), las empresas con ánimo de lucro pueden abarcar los aspectos de carácter social y medioambiental que predominan en las organizaciones no lucrativas y estas, a su vez, asegurarse la viabilidad económica necesaria para seguir operando.

Por último, con respecto al impacto social que el acuerdo de cooperación Grameen Danen Foods ha supuesto para la comunidad, se encuentran los siguientes beneficios. Por una parte, la planta situada en Bogra fabrica 100.000 unidades de yogur diariamente, lo que significa que 100.000 niños y niñas de la zona reciben un alimento que cubre el $30 \%$ de sus necesidades diarias de vitamina A, hierro, iodo y zinc (Danone Communities, 2020). De este modo, se consigue cumplir con el principal propósito por el que ambas entidades establecieron esta alianza: mejorar las condiciones nutricionales de los niños y niñas de Bangladesh, especialmente de aquellos que se encuentran en las zonas rurales.

Por otra parte, la coalición ha colaborado de un modo firme en la erradicación de la exclusión de los colectivos más empobrecidos a través de la creación de un gran número de empleos locales. Esta creación de empleos ha favorecido el acceso de los habitantes de las zonas rurales a bienes y servicios que anteriormente quedaban fuera de su alcance, como pueden ser el acceso a financiación, redes de agua potable o electricidad. Aspectos que han propiciado una importante reducción en la desigualdad de la población rural con respecto a las zonas más desarrolladas.

Por tanto, puede concluirse que, a través de este modelo de cooperación, citando una vez más el estudio realizado por Abenoza et al., (2015), las organizaciones implicadas, gracias a la puesta en común de sus diferentes recursos, son capaces de resolver un problema social de una forma mucho más eficiente de lo que podrían hacerlo en el caso de operar por separado. La empresa social Grameen Danone Foods, al producir y distribuir de forma local yogures a precios bajos asequibles por la población, posibilita una mejora en la alimentación y nutrición de los niños y a su vez, genera un ecosistema en el que intervienen diferentes actores de la comunidad; factor que les permite contar con una mayor capacidad económica, contribuyendo así a su progreso social y económico y al desarrollo de la región.

\subsection{Análisis comparativo de los dos casos estudiados}

A continuación, se realiza un análisis comparativo de las dos alianzas estratégicas estudiadas, con el propósito de 1) identificar las similitudes y diferencias entre los acuerdos de cooperación estudiados y 2) demostrar si tiene lugar o no, un patrón común en las alianzas estratégicas entre organizaciones lucrativas y sin ánimo de lucro. Con esta finalidad, se va a realizar un doble análisis: 1) un análisis comparativo de las razones de ser de ambos acuerdos de cooperación y 2) un análisis comparativo de sus implicaciones y beneficios.

\section{Razones de ser de las alianzas estratégicas}

Como se ha mencionado anteriormente, para ambos casos se encuentran una gran variedad de motivos que llevan a las diferentes organizaciones a llevar a cabo el proceso de alianza estratégica. En primer lugar, por un lado, en el caso de Grupo Vips-Fundación Hazlo Posible, se encuentra que, por parte de la entidad no lucrativa, el acuerdo de cooperación se establece con el propósito de acceder a una fuente de financiación privada para poder prosperar; mientras que, por parte de la organización con ánimo de lucro, la finalidad principal era el embarcarse en un proyecto de carácter social que le permitiera experimentar nuevas alternativas de crecimiento. Por otro lado, en el caso de Grameen-Danone Foods, la alianza se crea con el objetivo por parte de Grameen Bank de mejorar la alimentación de los niños y niñas más pobres de Bangladesh; mientras que el Grupo Danone ve una oportunidad de entrar en un nuevo mercado y de adquirir conocimientos y habilidades que le permitan desarrollar próximos proyectos sociales.

Por tanto, si bien los proyectos de cooperación difieren en lo que a la actividad a desarrollar se refiere (en el caso de Grupo Vips-Fundación Hazlo Posible la creación de un portal de voluntariado, mientras que en el caso de Grameen-Danone Foods, la producción y distribución de yogures nutritivos), en línea con el estudio de Contractor y Lorange (1988) mencionado en el marco teórico, puede observarse que ambas alianzas estratégicas coindicen en ser establecidas con la finalidad de, a través de la cooperación, acceder a nuevas capacidades, comportamientos complementarios y recursos necesarios de los que disponen los socios para poder desarrollar la actividad empresarial deseada de una manera óptima.

En segundo lugar, el acuerdo de cooperación Grupo Vips-Fundación Hazlo Posible ha facilitado la creación de una ventaja competitiva sostenible basada en la transferencia de conocimiento y confianza, tanto para la organización con ánimo de lucro como para la no lucrativa (Planellas y Urriolagoitia, 2011). Asimismo, se observa como a través de la alianza estratégica Grameen Bank-Danone Foods, la coalición se ha convertido en una fuente de capacidad distintiva a largo plazo sustentada en la creación de valor tanto 
social como económico (Fisac et al., 2011); consiguiendo de esta manera afianzar la supervivencia de las organizaciones. De este modo, queda de manifiesto como ambas alianzas estratégicas se establecen con el propósito mencionado por Arenas y García (2007), quienes afirman que los acuerdos de cooperación se llevan a cabo con la intención de establecer y mantener ventajas competitivas que permitan consolidar la longevidad de las organizaciones envueltas en el acuerdo.

Por tanto, tras el análisis realizado, puede concluirse que las razones de ser de las alianzas estratégicas Grupo Vips-Fundación Hazlo Posible y Grameen Bank-Danone Foods, siguen un patrón común, puesto que ambas se establecieron con la intención de adquirir nuevas capacidades a través de la transferencia de conocimientos y de crear a través de su actividad una ventaja competitiva sostenible. En la Figura 1 se recogen las distintas semejanzas y diferencias expuestas en este trabajo relativas a las razones de ser por las que se establecen las alianzas estratégicas de los dos casos estudiados.

Figura. 1. Compilación de las semejanzas y diferencias en las razones de ser de las alianzas estratégicas Grupo VipsFundación Hazlo Posible y Grameen-Danone Foods

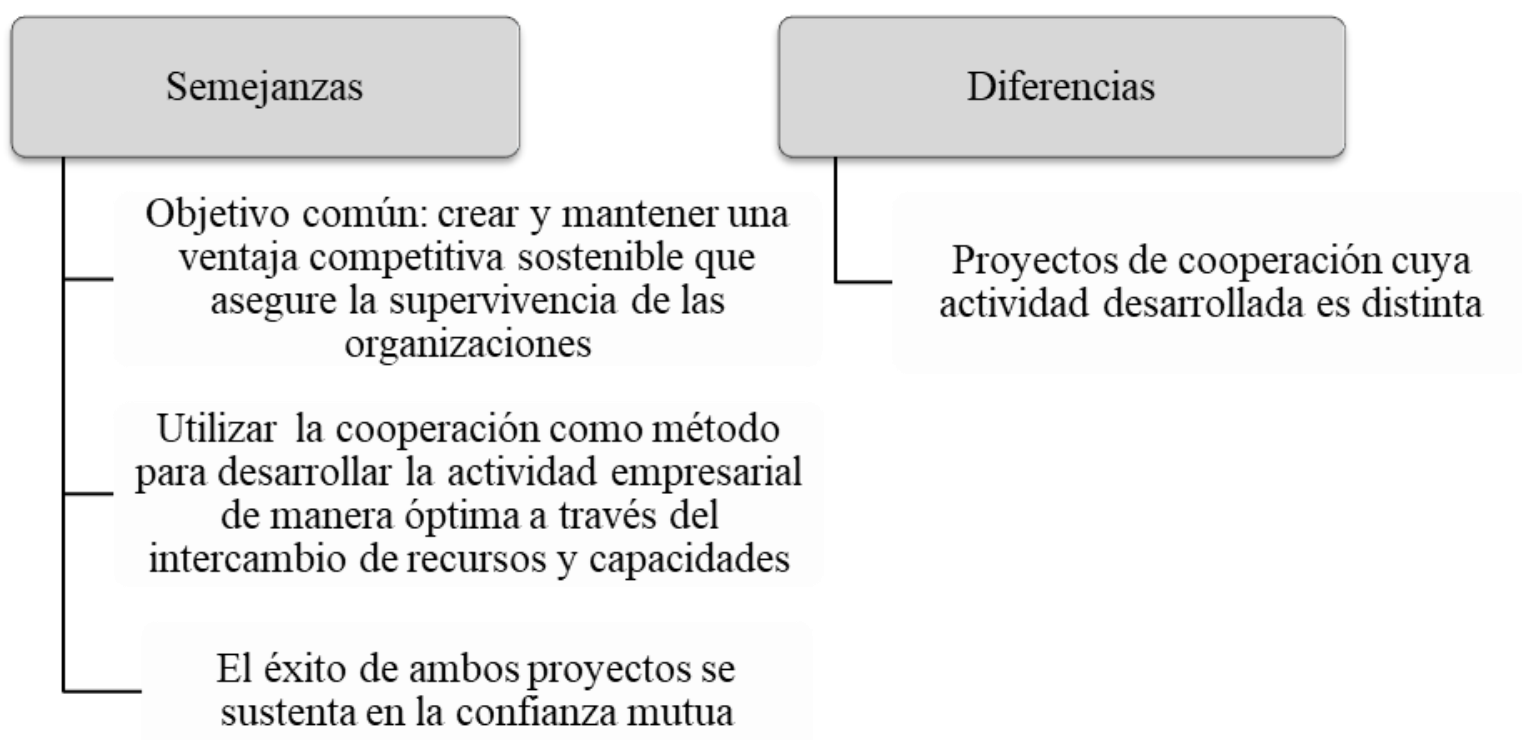

Fuente: Elaboración propia

\section{Implicaciones y beneficios de las alianzas estratégicas}

Como se ha mencionado anteriormente, para ambos casos se encuentran una gran variedad de implicaciones y beneficios tanto para las entidades cooperantes como para la comunidad, como resultado de los acuerdos de cooperación estratégicos.

En primer lugar, con lo que respecta a los beneficios proporcionados a las organizaciones, tanto Grupo Vips-Fundación Hazlo Posible como Grameen Bank-Danone Foods, debido a la transferencia de conocimientos y capacidades, han podido adquirir un know how único (Carreras e Iglesias, 2012), que ha dado lugar a una sinergia singular, valiosa e inimitable y, por tanto, a una ventaja competitiva sostenible (Arenas y García, 2007). Carreas e Iglesias (2012) apuntan que este know how ha permitido a ambas coaliciones beneficiarse a través del acceso a nuevos destinatarios, gracias a la creación de nuevos servicios (en el caso de Grupo Vips-Fundación Hazlo Posible, un portal web de voluntariado) o productos (como se observa en el caso de Grameen Bank-Danone Foods con la producción y distribución de yogures nutritivos). Asimismo, los autores también indican que gracias a las alianzas estratégicas entre entidades no lucrativas y con ánimo de lucro, estas últimas experimentan una mayor facilidad a la hora de implementar políticas de responsabilidad social corporativa; aspecto que se observa en el caso de Danone, puesto que a raíz de la alianza con el Banco Grameen, la compañía ha podido implementar diversas políticas de responsabilidad social en sus diversas filiales alrededor del mundo (Danone Communities, 2020b).

En segundo lugar, en relación a la teoría expuesta anteriormente en el trabajo de Rodríguez et al. (2013), al afirmar que las entidades se benefician de una mayor capacitación de nuevos socios, cabe destacar que ambos tipos de organización han experimentado un importante aumento en su número de socios. Tanto Grupo Vips y Danone, como Fundación Hazlo Posible y Banco Grameen han alcanzado una mayor visibilidad ante la comunidad gracias a la alianza, por lo que sus redes se han visto gratamente ampliadas. Del mismo modo, Rodríguez et al. (2013) mencionan una relación positiva entre la reputación corporativa de 
la entidad lucrativa y la cooperación entre organizaciones con ánimo y sin ánimo de lucro. Tras el estudio empírico realizado, puede afirmarse que esta teoría concuerda con la realidad de las empresas estudiadas: Grupo Vips, desde el establecimiento del acuerdo de cooperación, es considerada como una de las empresas más reputadas de su sector (Grupo Vips, 2020b); de igual modo que Danone Foods, gracias a la creación de Danone Communities a raíz de la alianza con el Banco Grameen, ha sido considerada en el período comprendido entre los años 2010-2015 como una de las organizaciones más reputadas de su sector (Corporate Danone, 2020b).

En tercer lugar, en lo relacionado al beneficio aportado a la comunidad como consecuencia de este tipo de alianza estratégica, tras el análisis realizado de los dos acuerdos de cooperación, se encuentra también en este aspecto un patrón común entre las coaliciones: ambas consiguen la resolución de un problema de carácter social a través de la puesta en común de sus diferentes recursos. Por un lado, Grupo Vips (aportando financiación y contactos), y la Fundación Hazlo Posible (contribuyendo con sus conocimientos en materia de responsabilidad social y aspectos de carácter no lucrativo), consiguen expandir un portal web destinado a la inserción de empresas y diferentes agentes de la sociedad en cuestiones de voluntariado. De esta manera, puede observarse que en línea con el estudio mencionado en el marco teórico de Abenoza et al., (2015), Grupo Vips-Fundación Hazlo Posible consiguen resolver un problema comunitario a través de la innovación social, esto es, las empresas contribuyen al desarrollo tecnológico a través de su portal de Internet, tecnología que como se ha demostrado a lo largo del trabajo tuvo una importante repercusión y beneficio social.

En la misma línea, Danone Foods, al contribuir con su know how en materia de elaboración de productos alimenticios, en conjunto con el conocimiento sobre el mercado de Bangladesh y funcionamiento de las empresas sociales aportado por el Banco Grameen, logran mejorar la alimentación de los niños y niñas de la ciudad, así como el progreso económico del área en la que se establecen gracias a la creación de empleo. De esta manera, puede concluirse que los acuerdos de cooperación estudiados coinciden en que ambos permiten la resolución de un problema de carácter social; implicación que no hubiese tenido lugar en el caso de operar por separado (Abenoza et al., 2015).

Por último, cabe destacar, que si bien ha sido a través de la innovación social (como en el caso de la alianza Grupo Vips-Fundación Hazlo Posible) o del progreso económico (Grameen Bank-Danone Foods), ambos acuerdos de cooperación han dado lugar a la creación de valor compartido referida por Porter y Kramer (2011). Ambas alianzas estratégicas han conseguido, a través de su actividad, generar un beneficio social que, a su vez, les ha permitido originar beneficio económico. Como consecuencia de su trabajo conjunto, las organizaciones implicadas en el acuerdo de colaboración logran redefinir la idea concebida de entidad lucrativa o entidad sin ánimo de lucro, convirtiéndose en una organización de carácter híbrido que asegura su viabilidad económica y supervivencia mediante el avance simultáneo de la competitividad empresarial, su entorno económico y social (Porter y Kramer, 2011).

En la Figura 2 se recogen las distintas semejanzas expuestas en este trabajo relativas a las implicaciones y beneficios reportados por las alianzas estratégicas Grupo Vips-Fundación Hazlo Posible y Grameen BankDanone Foods.

Figura. 2. Compilación de las semejanzas en las implicaciones y beneficios de las alianzas estratégicas Grupo VipsFundación Hazlo Posible y Grameen-Danone Foods

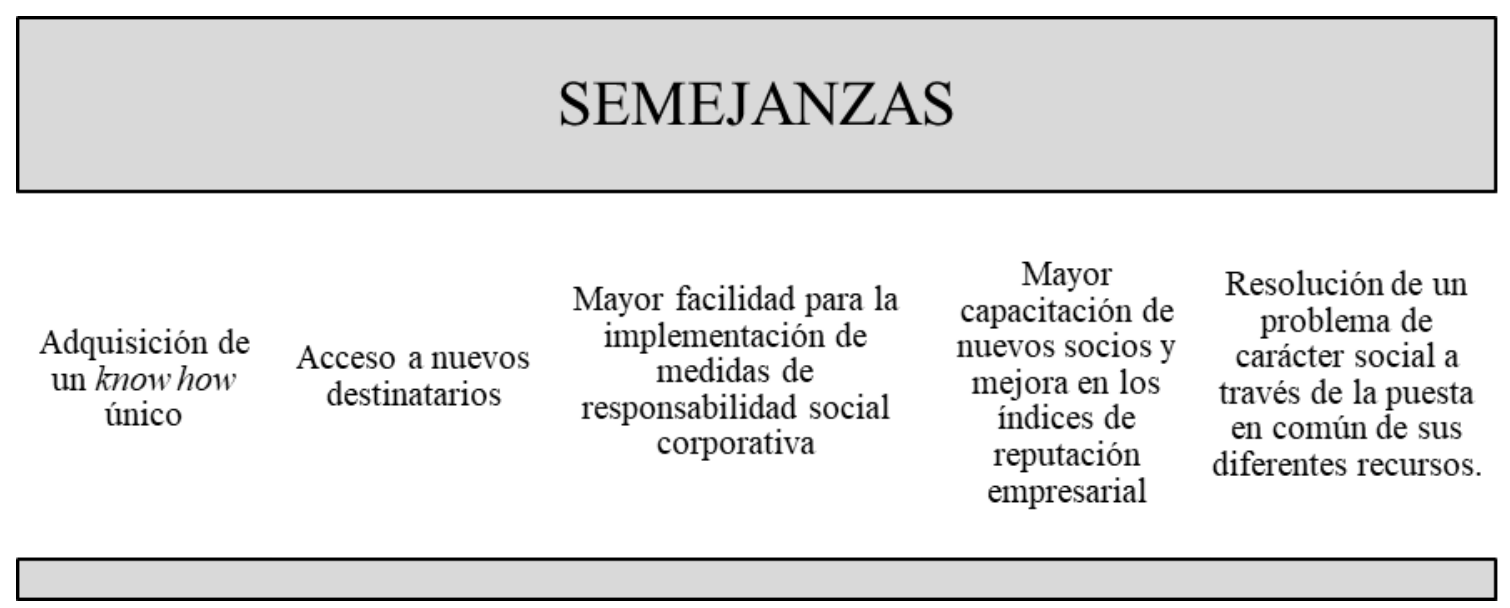

Fuente: Elaboración propia

Por todo ello, basado en el análisis comparativo sobre las razones de ser y beneficios de las alianzas estratégicas Grupo Vips-Fundación Hazlo Posible y Grameen Bank-Danone Foods, puede concluirse que no existen diferencias significativas entre los casos y, por tanto, puede afirmarse que tiene lugar un patrón 
común en ambos acuerdos de cooperación en cuanto a los aspectos estudiados se refiere. Ambos proyectos comparten tanto los motivos por los que son creados, como los beneficios que suponen para las organizaciones y los diferentes agentes de la sociedad. Uno y otro muestran como resulta clave para el éxito en la cooperación la confianza mutua, de modo que la transferencia de conocimiento, así como la puesta en común de recursos y esfuerzos sea prolongada en el tiempo. De esta manera, se generan ganancias económicas que al reinvertirse en la organización posibilitan seguir con la actividad de carácter social, prolongando así los beneficios tanto en la esfera social como en la económica.

\section{Conclusiones}

El entorno actual en el que vivimos exige a las organizaciones actitudes más sostenibles; la sociedad demanda que las actuaciones y desarrollo empresarial no solo permitan la viabilidad económica, sino también la social y medioambiental y para ello, es necesario que la ética y responsabilidad social tengan cabida en las organizaciones. Es a través de comportamientos éticos y socialmente responsables cuando las entidades son capaces de crear valor tanto para ellas como para sus diferentes stakeholders, de manera que estas conductas se han convertido en necesarias para asegurar la supervivencia y crecimiento de las organizaciones en el siglo XXI.

Si bien la ética y responsabilidad social son aspectos indispensables para garantizar el éxito de las organizaciones actuales, existe un tercer elemento cuya importancia también ha ido en aumento en las últimas décadas: la cooperación. En los últimos años ha tenido lugar un claro aumento en el número de alianzas estratégicas entre organizaciones como consecuencia de los diversos beneficios, expuestos a lo largo de este trabajo, que estas reportan tanto a las empresas como a los diferentes agentes de la sociedad. Sin embargo, el simple hecho de cooperar no es indicativo de creación de valor; se debe considerar la importancia que los comportamientos éticos y socialmente responsables tienen en el éxito de la cooperación.

Para poder alcanzar los objetivos comunes por los que se llevan a cabo alianzas entre organizaciones y que estas sean sostenibles en el tiempo, es imprescindible que se establezca un clima de confianza mutua entre los socios. Confianza que solo será posible si todas las partes basan sus actuaciones en comportamientos éticos que permitan aportaciones sinceras y generosas a los acuerdos. Por tanto, la importancia de la ética en los acuerdos de cooperación reside en que ésta permite el establecimiento de un clima de confianza que será indispensable para el éxito de la alianza. Como consecuencia de esta ética cooperativista indispensable para el éxito de las alianzas y con la intención de dar respuesta a los requerimientos que demanda la sociedad actual a las empresas, éstas son capaces de desarrollar conjuntamente políticas de responsabilidad social destinadas a la creación de valor compartido, valor que no queda limitado al beneficio propio de los socios, sino al de los diferentes agentes de la comunidad.

El aumento de la demanda social de este valor compartido en las últimas décadas, ha llevado a las organizaciones a realizar acuerdos de cooperación con entidades cuyo fin es distinto; como se ha demostrado a lo largo de este trabajo, a través de las alianzas estratégicas entre organizaciones lucrativas y sin ánimo de lucro, las entidades son capaces de acceder a los recursos y capacidades clave para la implementación de medidas de responsabilidad social que permiten la creación de valor compartido; valor basado en el desarrollo económico a través del desarrollo social y viceversa.

Como se ha observado en el estudio empírico, este valor compartido tiene diversas implicaciones reales para las organizaciones y la sociedad en su conjunto. En primer lugar, con lo que respecta a los beneficios para las organizaciones lucrativas, tanto Grupo Vips como Danone se han visto beneficiados de una mayor imagen y reputación corporativa gracias a sus alianzas con las entidades de carácter social estudiadas. Asimismo, la experiencia y conocimientos adquiridos a través del intercambio de recursos y capacidades, les ha permitido a ambas entidades con ánimo de lucro contar con una mayor facilidad para la implementación de medidas de responsabilidad social corporativa independientes a la alianza, así como con un mayor número de socios, a raíz de una mayor visibilidad y reputación.

En segundo lugar, las organizaciones sin ánimo de lucro Fundación Hazlo Posible y Grameen Bank, se han visto beneficiadas, por un lado, del acceso a recursos económicos, factor que les ha permitido desarrollar su actividad con un mayor alcance social, accediendo de esta manera a un número superior de destinatarios. Por otro lado, ambas han experimentado un aumento en su número de socios como consecuencia de la alianza, puesto que, a través de ésta, se han favorecido de una mayor visibilidad.

En tercer lugar, el estudio empírico realizado en este trabajo demuestra que este tipo de alianzas estratégicas también reporta beneficios para las organizaciones socias como conjunto; gracias a los acuerdos de cooperación Grupo Vips-Fundación Hazlo Posible y Grameen Bank-Danone Foods, las entidades, a través de la transferencia de recursos, capacidades y conocimientos, han generado un know how único que les ha permitido la creación de una ventaja competitiva sostenible.

Esta ventaja competitiva sostenible no reporta únicamente beneficios para las organizaciones, sino también para la comunidad. Ambos casos estudiados coinciden en haber propiciado la mejora y solución de 
un problema de carácter social a través de la alianza. Por un lado, Grupo Vips-Fundación Hazlo Posible consigue crear una red de voluntariado corporativo destinada a favorecer la cultura del voluntariado y ayuda social en España; y por otro lado, Grameen Bank-Danone Foods asiste a la erradicación de la desnutrición infantil y desempleo en las zonas más empobrecidas de Bangladesh. De este modo, se observa como ambos acuerdos consiguen entrelazar la creación de valor económico y social: el estudio comparativo realizado en este trabajo demuestra como a través de la cooperación entre entidades con ánimo de lucro y entidades no lucrativas, las organizaciones son capaces de crear valor compartido, esto es, generar un beneficio social que a su vez propicie beneficio económico, el cual, al reinvertirse en la organización permite perpetuar el impacto positivo en la comunidad.

El estudio comparativo realizado pone de manifiesto como las alianzas estratégicas Grupo VipsFundación Hazlo Posible y Grameen Bank-Danone Foods, acuerdos basados en la persecución de un objetivo común y la confianza, han demostrado con los años de trabajo conjunto, que a través de la creación de valor compartido las entidades son capaces de disgregar las diferencias en cuanto a sus fines se refiere, convirtiéndose de esta manera en organizaciones de carácter hibrido, que al incluir las necesidades y demandas de todos los grupos de interés o stakeholders y contar con los diferentes recursos y capacidades necesarias para hacer frente a estas, consiguen asegurar su longevidad como organización.

Si bien los resultados del estudio empírico de este trabajo coinciden con las teorías expuestas en el marco teórico en relación con los motivos por los que las organizaciones lucrativas y sin ánimo de lucro llevan a cabo alianzas estratégicas, así como sus implicaciones y beneficios, este trabajo se limita al estudio de dos acuerdos de cooperación, por lo que las conclusiones obtenidas solo son aplicables y comparables a estos casos. Con el propósito de verificar si los resultados obtenidos en este trabajo son extrapolables o no, debería llevarse a cabo la realización de un estudio empírico cuantitativo o una ampliación del estudio cualitativo a un mayor número de casos.

\section{Referencias bibliográficas}

Abenoza, S., Carreras, I., y Sureda, M. (2015) Colaboraciones ONG y empresa que transforman la sociedad. Programa ESADE-PwC de Liderazgo Social 2014-15, Instituto de Innovación Social, Universidad Ramón Llull.

Altamira, R. (2000) La negociación de alianzas estratégicas internacionales, Revista de Dirección, Organización y Administración de Empresas, $\mathrm{N}^{\circ}$ 24, pp. 99-106.

Al-Tabbaa, O., Leach, D., y March, J. (2014) Collaboration between nonprofit and business sectors: a framework to guide strategy development for nonprofit organizations, Voluntas, Vol. 25, $\mathrm{N}^{\mathrm{o}} 3$, pp. 657-678.

Amran, A., Lee, S. P., y Devi, S. S. (2014) The influence of governance structure and strategic corporate social responsibility toward sustainability reporting quality, Business Strategy and The Environment, Vol. 23, $\mathrm{N}^{\circ}$ 4, pp. 217-235.

Arenas, J., y García, R. (2007) Las alianzas estratégicas internacionales el impacto del origen del socio en la relación, Revista de Estudios Empresariales. Segunda época, $\mathrm{N}^{\circ}$ 1, pp.73-89.

Arya, B., y Salk, J. E. (2006) Cross-sector alliance learning and effectiveness of voluntary codes of corporate social responsibility, Business Ethics Quarterly, Vol. 16, № 2, pp. 211-234.

Barrera, E. (2007) La empresa social y su responsabilidad social, Innovar, Vol. 17, No 30, pp. 59-76.

Berger, I. E., Cunningham, P. H., y Drumwright, M. E. (2004) Social alliances: company/nonprofit collaboration, California Management Review, Vol. 47, № 1, pp. 58-65.

Browning, L., Beyer, J. M., y Shetler, J. C. (1995) Building cooperation in a competitive industry: Sematech and the semiconductor industry, The Academy of Management Journal, Vol. 38, № 1, pp. 113-151.

Callejas, R. J. M., y Salazar, J. (2012) La Alianza Universidad-Empresa-Estado y los procesos de I+ D: una mirada al caso antioqueño, Perfil de Coyuntura Económica, № 19, pp. 157-176.

Canzaniello, A., Hartmann, E., y Fifka, M. S. (2017) Intra-industry strategic alliances for managing sustainability related supplier risks motivation and outcome, International Journal of Physical Distribution \& Logistics, Vol. 47, $\mathrm{N}^{\circ}$ 5, pp. 387-409.

Carreras, I., y Iglesias, M. (2012) Modelo de J. Austin sobre alianzas sociales: La colaboración efectiva de las ONG. ESADE. Instituto de Innovación Social, p. 96.

Contractor, F. J., y Lorange, P. (1988) Why should firms cooperate? The strategy and economics basis for cooperative ventures, Cooperative Strategies in International Business, $\mathrm{N}^{\mathrm{o}}$ 1, pp 3-30.

Corporate Danone (2020a) Danone Communities. Disponible http://corporate.danone.es/es/descubre/sostenibilidad/un-enfoque-empresarialunico/danonecommunities/\#.XorzP4gzbIU.

Corporate Danone (2020b) Avales y Premios. Disponible en: https://www.danone.es/es/densia/avales-premios.html.

Danone (2020) Sobre nosotros: Creación de valor sostenible. Mirando hacia atrás en un viaje épico. Danone: One Planet, One Health. Disponible en: https://www.danone.com/about-danone/Danone-yogurt-turns-100/looking-backat-an-epic-journey.html. 
Danone Communities (2020a) Grameen Danone Foods Ltd. Disponible en: https://www.danonecommunities.com/grameen-danone-foods-ltd/.

Danone Communities (2020b) We alleviate poverty: We grow inclusive business solutions. Disponible en: http://www.danonecommunities.com/.

Díaz Gamarra, M. (2018) La transferencia de conocimiento en las alianzas estratégicas para la innovación, Revista de Ciencias de la Gestión, № 3, pp.136-148.

Fernández, M. C. (1999) Alianzas estratégicas de carácter tecnológico, Economía Industrial, Nº 330, pp. 31-42.

Fisac, R., Moreno, A., Mataix, C., y Palacios, M. (2011) Emprendimiento Social, Revista Española del Tercer Sector, $\mathrm{N}^{\mathrm{o}} 17$, pp. 55-62.

Fundación Seres (2020) Grameen - DANONE Foods Limited. Fundación Sociedad y Empresa Responsable. Disponible en: https://www.fundacionseres.org/Publicaciones/proyectos-colaboracion-entidad/grameen-danone-foods-limited/.

Gaona, M. B., Rojas, L. P., y Ramírez, A. J. F. (2019) Aplicación del conocimiento como bien común y mejora social, Mercados y Negocios, $\mathrm{N}^{\circ} 39$, pp. 131-160.

García, C., Raufflet, E., y Portales, L. (2011) Las alianzas entre empresas y ONG como medio para promover la RSE y el desarrollo local, Innovation RICEC, Vol. 3, No 1, pp. 1-15.

Grameen Bank (2020) Introduction. Disponible en: https://grameenbank.org/introduction/.

Grameen Foundation (2020) Our family of organizations. Disponible en:https://grameenfoundation.org/about-us/thegrameen-family.

Grupo Vips (2020a) Datos Clave. Disponible en: https://www.grupovips.com/quienes-somos.

Grupo Vips (2020b) Hacemos Comunidad. El Voluntariado Corporativo es parte de nuestro ADN. Disponible en: https://www.grupovips.com/quienes-somos/responsabilidad-corporativa/hacemos-comunidad.

Hansen, E. G., Sextl. M., y Reichwald, R. (2010) Managing strategic alliances through a community-enabled Balanced Scorecard: the case of Merck Ltd., Thailand, Business Strategy and the Environment, Vol. 19, No 6, pp. 387-399.

Harrison, J. S., y Berman, S. L. (2016) Corporate Social Performance and Economic Cycles, Journal of Business Ethics, Vol. 138, No 2, pp. 279-294.

Hazlo Posible (2019) Talento que Impacta. Disponible en: https://www.hazloposible.org/wpcontent/uploads/2019/03/Red-Talento-que-Impacta-Empresas-2019.pdf.

Hazlo Posible (2020) Alianzas y colaboraciones. Disponible en: https://www.hazloposible.org/alianzas-ycolaboraciones/.

Im, J. (2011) The role of NGO partner's knowledge and reputation on corporate learning in cross-sector alliances, Journal of Strategic Management, Vol. 14, N 3, pp. 67-88.

Kliksberg, B. (2006) Ética empresarial: ¿moda o demanda imparable?. Formación Carolina. Buenos Aires: Universidad de Buenos Aires.

Kopka, K., Mahoney, L. S., Convery, S. P., y LaGore, W. (2014) An examination of alliances and corporate social responsibility, Research on Professional Responsibility and Ethics in Accounting, № 18, pp. 109-130.

Lai, J. H., y Chen, L. Y. (2014) The valuation effect of corporate governance on stakeholder wealth: evidence fron strategic alliances, International Review of Economics \& Finance, $\mathrm{N}^{\circ} 32$, pp. 117-131.

Lajara, B. M., Lillo, F. G., y Ramón, D. Q. (2003) Factores de éxito y fracaso de la cooperación entre empresas: un enfoque organizativo y estratégico,Dirección y Organización: Revista de Dirección, Organización y Administración de Empresas, № 29, pp. 1-11.

Liu, G., y Ko, W. W. (2011) An analysis of cause-related marketing implementation strategies through social alliance: partnership conditions and strategic objectives, Journal of Business Ethics, Vol. 100, No 2, pp. 253-281.

Ma,Y. Y., Sia, C. L., Li, Y. et al. (2016) Sources of resources, alliance green management, and alliance performance in an emerging economy, Journal of Cleaner Production, No 139, pp. 319-327.

Michavila, F. (2011) Del aislacionismo a las alianzas, Arbor: ciencia, pensamiento y cultura, Vol. 187, № 3, pp. 67-75.

Mio, C. (2010) Corporate social reporting in Italian multi-utility companies: An empirical analysis, Corporate Social Responsibility and Environmental Management, Vol. 17, № 5, pp. 247-271.

Morales, H. N., González, J. G., y Torres, M. A. (2011) La creación de valor compartido y la innovación social como factores de sinergia para el desarrollo económico, Instituto de Ciencias Básicas e Ingeniería, № 1, pp. 1-19.

Muñoz Martín, J., y Montoro Sánchez, M. (2007) Enfoques teóricos para el estudio de la cooperación empresarial, Cuadernos de Estudios Empresariales, $\mathrm{N}^{\circ}$ 17, pp. 141-163.

Muthuri, J. N., Chapple, W., y Moon, J. (2009) An Integrated Approach to Implementing Community Participation in Corporate Community Involvement: Lessons from Magadi Soda Company in Kenya, Journal of Business Ethics, Vol. 85, № 2, pp. 431-444.

Norheim-Hansen, A. (2013) A natural resource based examination of strategic alliance formation. Interpartner Dynamics in Strategic Alliances, Book series Research in Strategic Alliances, No 1, pp. 73-96.

Pérez López, J.A. (1993) Fundamentos de la Dirección de Empresas, Madrid: Ediciones Rialp.

Planellas, M., y Urriolagoitia, L. (2011) La alianza estratégica entre Grupo Vips y Fundación Hazlo Posible. Un ejemplo de colaboración empresa-ONG, Barcelona: ESADE, Instituto de Innovación Social, Universidad Ramón Llull.

Porter, M. E., y Kramer, M. R. (2011) Creating shared value. How to Reinvent Capitalism—and Unleash a Wave of Innovation and Growth, Harvard Business Review, Vol. 89, No 1-2, pp. 62-77. 
Prado-Lorenzo, J. M., Gallego-Alvarez, I., Garcia-Sanchez, I. M. (2009) Stakeholders engagement and corporate social responsibility: The ownership structure effect, Corporate Social Responsibility and Environmental Management, Vol. 16, No 2, pp. 94-107.

Pulgar, C., y Pelekais, C. (2014) Responsabilidad social basada en alianzas estratégicas en universidades de gestión privada, Revista de Formación General, Vol. 13, № 2, pp.199-220.

Radrigán, M. H., Dávila, A. M., y Penaglia Vasquez, F. E. (2012) Gestión y liderazgos en los emprendimientos sociales: el caso del sector no lucrativo chileno, Polis. Revista Latinoamericana, № 32, pp. 1-15.

Renart Cava, L. G. (1999) Consorcios de exportación y otros tipos de alianzas estratégicas entre empresas, Boletín económico de ICE, No 2627, pp. 1001-1016.

Reptrak (2020) What is your company reputation?. Disponible en: https://www.reptrak.com/blog/.

Rim, H., Yang, S. U., y Lee, J. (2016) Strategic partnerships with nonprofits in corporate social responsibility CSR: the mediating role of perceived altruism and organizational identification, Journal of Business Research, Vol. 69, № 9, pp. 3213-3219.

Rivera-Santos, M., y Rufin, C. (2010) Odd couples: inderstanding the governance of firm-NGO alliances, Journal of Business Ethics, № 94, pp. 55-70.

Rodríguez, D., Flores, R., y Miranda, P. (2013) Alianzas entre empresas lucrativas y organizaciones sin fines de lucro (OSFL): Estudios de casos en chile, Universum (talca), Vol. 28, No 1, pp. 173-202.

Romero, A. M., y Oliva, E. J. D. (2012) Formas y categorización de las relaciones entre las organizaciones sin fines de lucro y el sector privado: una revisión, Administración y Desarrollo, Vol. 40, N 55, pp. 77-92.

Rueda López, R. (2016) La ética como fundamento de la responsabilidad social: el fomento de la perspectiva de género como praxis socialmente responsable en la universidad. Córdoba: Tesis Doctoral, Universidad de Córdoba.

Rule, P., y John, V. M. (2015) A necessary dialogue: Theory in case study research, International Journal of Qualitative Methods, Vol. 14, No 4, pp. 1-11.

Shumate, M., y O'Connor, A. (2010) Corporate reporting of cross-sector alliances: the portfolio of NGO partners communicated on corporate websites, Communication Monographs, Vol. 77, № 2, pp. 207-230.

Silvestri, A., y Veltri, S. (2017) Ethical strategic alliances for the sustainable recovering of a territory, Corporate Governance. The International Journal of Business in Society, Vol. 17, № 2, pp. 230-249.

Thorne, L., Mahoney, L. S., y Gregory, K. (2017) A comparison of Canadian and US CSR Estrategic alliances, CSR Reporting and CSR Performance: insights into implicit-explicit CSR, Journal of Business Ethics, Vol. 143, N 1, pp. 85-98.

Van Der Voort, J. M., Glac, K., y Meijs, L. C. (2009) 'Managing' corporate community involvement. Journal of Business Ethics, Vol. 90, N 3, pp. 311-329.

Yin, R. K. (2002) Case study research: Design methods. Thousand Oaks, CA: SAGE Publications.

Yin, R. K. (2013) Validity and generalization in future case study evaluations, Evaluation, Vol. 19, № 3, pp. 321-332. 